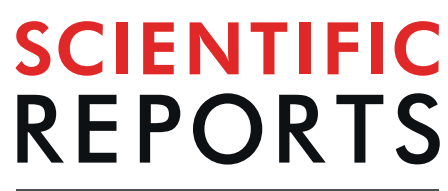

natureresearch

\title{
A novel shaped-controlled fabrication of nanopore and its applications in quantum electronics
}

\begin{abstract}
Chien-Han Chen ${ }^{1}$, Xuyan Chang ${ }^{2} \&$ Cen-Shawn Wu ${ }^{1,2^{*}}$
High-intensity $\left(10^{7}-10^{8} \mathrm{~A} \mathrm{~m}^{-2}\right)$ electron beams can be used to fabricate nanoscale pores. This approach enables real-time observation of nanopore drilling and precise control of the diameter of the nanopore. Nevertheless, it is not suitable for tuning the nanopore's sidewall shape. In this study, we demonstrate the use of low-intensity electron beams to fabricate nanopores on a silicon nitride $\left(\mathrm{SiN}_{\mathrm{x}}\right)$ membrane. This technique allows the precise adjustment of the nanopore dimension and the shaping of its three-dimensional (3D) nanostructure. The 3D structures of the nanopore were evaluated by electron tomography, and series of oblique images were used in reconstructing the 3D images of nanopores using a weighted back-projection method. The sidewall shape of the nanopore was observed at different electron-beam conditions, and the formation mechanism was elucidated based on these results. The nanopore fabricated with this technique can be used as a template to develop electronics at the nanoscale based on which a quantum-dot device can be prepared with a simple evaporation process. The measured results show that the device can resolve well-defined electronic states that are characteristic for the behaviors of the quantum-dot device.
\end{abstract}

In the past decade, solid-state nanopores have drawn extensive attention because of their capacity for use for rapid electronic signal and biomolecular detections, and for the tremendous prospect for use in the next generation of diagnostic devices in nanomedicine ${ }^{1-11}$. The reactive ion etching technology $y^{12}$ or the electron beam from a transmission electron microscope (TEM) can be used to construct nanopores on silicon nitride $\left(\mathrm{SiN}_{\mathrm{x}}\right)^{13,14}$ and silicon oxide $\left(\mathrm{SiO}_{2}\right)$ membranes ${ }^{15}$. These two approaches demonstrate great repeatability and have been widely used in fabricating nanopores, but the method of choice is to drill them using an electron beam in a TEM ${ }^{16,17}$. The advantage of the use of an electron beam to make nanopores is that a) it can precisely control the size of the nanopore and b) it does not cause ionic contamination. These advantages are increasingly important as nanopores are used in the system of biomolecular detections. In the study of electron-beam drilling by Dekker et al. ${ }^{18}$, a high-intensity focused electron beam was used to drill nanopores on a silicon oxide membrane. This showed that the electron beam could be accurately controlled for drilling pores at the nanometer scale while the drilling condition was monitored.

In deoxyribonucleic acid (DNA) sequencing, the size of the nanopore is important ${ }^{19,20}$, whereas the sidewall shape constitutes another critical factor ${ }^{21}$. In this study, we used a low-intensity electron beam to create nanopores on a $\mathrm{SiN}_{\mathrm{x}}$ membrane. This technique allows the precise adjustment of the nanopore dimensions, and creates its three-dimensional (3D) shape. The electron tomography is a powerful technique which has been used to characterize $3 \mathrm{D}$ nanoscale structures ${ }^{12,22}$. Accordingly, in this study, we investigated that the mechanism of nanopore sculpting with low intensity e-beam by employing electron tomography, and characterizing the drilling behavior of nanopores. Finally, we introduced a new manufacturing technology to prepare a metallic quantum-dot device. This device used the reconstructed nanopore as a template to deposit metal on its anterior and posterior sides to form a sandwich structure of the metallic nanopore. The measured results show that the device can be defined as a quantum-dot device with a discrete energy level that can be clearly observed.

\section{Experimental Details}

The growth of low-stress amorphous silicon nitride membranes (thicknesses of 30, 75, and $110 \mathrm{~nm}$ ) was carried out using the low-pressure chemical-vapor-deposition (LPCVD) method on a substrate with a thickness of $500 \mu \mathrm{m}$ at a temperature of $780^{\circ} \mathrm{C}$ immersed in ammonia and exposed to dichlorosilane gases. The thickness tolerance of the prepared membrane was approximately $10 \%$ and was measured by spectroscopic ellipsometry.

${ }^{1}$ Graduate Institute of Photonics, National Changhua University of Education, Changhua, 500, Taiwan. ${ }^{2}$ Department of Physics, National Changhua University of Education, Changhua, 500, Taiwan. *email: wucs@cc.ncue.edu.tw 
(a)

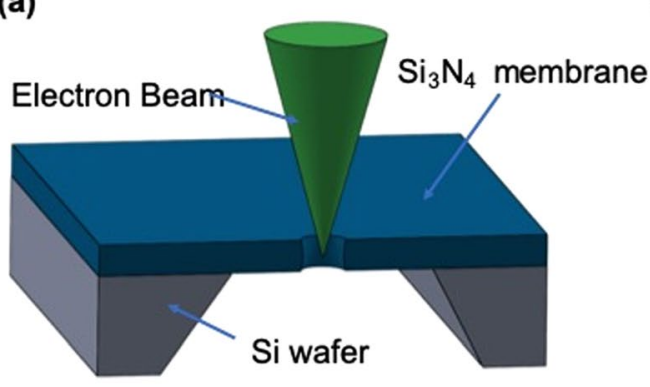

(c)

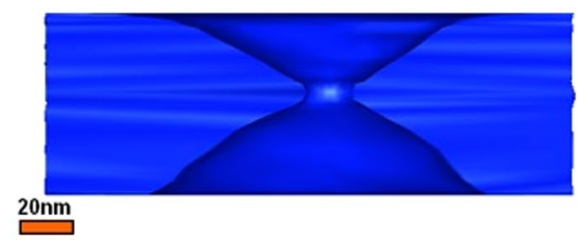

(b)

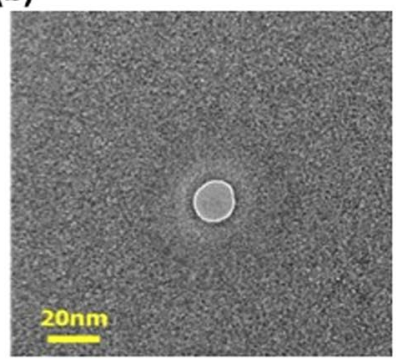

(d)

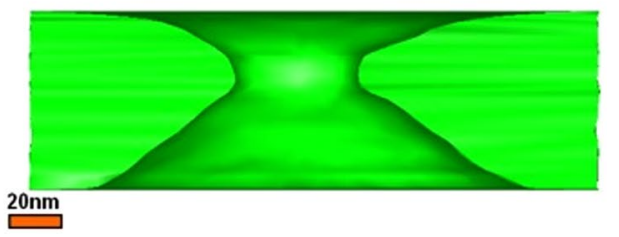

Figure 1. (a) Schematic of the drilling process of the nanopore with the use of a focused electron beam. (b) Transmission electron microscopy (TEM) image of a nanopore with a diameter of $\sim 15 \mathrm{~nm}$ in $\mathrm{SiN}_{\mathrm{x}}$ membranes with thicknesses of $75 \mathrm{~nm}$. (c) Reconstructed three-dimensional (3D) image of nanopore with a diameter of $\sim 15 \mathrm{~nm}$ in $\mathrm{SiN}_{\mathrm{x}}$ membranes with thicknesses of $75 \mathrm{~nm}$. Tomographic reconstruction is calculated by weighted back projection. The shape of the sidewall shape is hourglass. (d) 3D image of a nanopore with a diameter of $\sim 50 \mathrm{~nm}$ in $\mathrm{SiN}_{\mathrm{x}}$ membranes with a thickness of $75 \mathrm{~nm}$.

Thereafter, photolithography and reactive ion etching (RIE) were used to form a square window with a side length of $750 \mu \mathrm{m}$ on the silicon nitride membrane. This window was used as a mask for $\mathrm{KOH}$ wet etching. Given that the $\mathrm{KOH}$ was anisotropically etched on the silicon substrate, a silicon substrate with a pyramidal shape was formed. A square suspension of the silicon nitride membrane with a side length of approximately $30 \mu \mathrm{m}$ was obtained on the silicon substrate notch.

A low-intensity electron beam $\left(\sim 10^{6} \mathrm{~A} \mathrm{~m}^{-2}\right)$ from a Jeol 2010 TEM with an acceleration voltage of $200 \mathrm{keV}$ was used to drill the nanopore on the silicon nitride membrane while the entire drilling process was monitored. The focused electron beam diameter can be defined by the spot size on the membrane upper surface, which had a diameter of approximately $3-5 \mathrm{~nm}$. The nanopore drilling time was approximately $1-5$ minutes, depending on the thickness of the membrane. TEM images can provide real-time feedback and allow control during the nanopore drilling processes. With in-situ electron microscopy, 2D imaging provides instant feedback for pore drilling. By using real- time image feedback, we could precisely and repeatedly manufacture the sidewall shape of the nanopore. In addition, we demonstrated the use of the low-intensity electron beam to fabricate nanoscale devices of arbitrary geometries on insulating platforms without the need for a mask or resist (see the Figs. S1-S3 of Supplementary Material). The IMOD software modeling program was used to reconstruct images to facilitate the evaluation of the $3 \mathrm{D}$ reconstructed images ${ }^{23,24}$. The shape of nanopore can be depicted and analyzed by $3 \mathrm{D}$ TEM tomography (see the Fig. S4 of Supplementary Material). Before reconstructing the 3D structure of the nanopore, a TEM equipped with a Gatan Ultrascan $2.4 \mathrm{k} \times 4 \mathrm{k}$ charge-coupled device $(\mathrm{CCD})$ camera was used to acquire a series of oblique images of the nanopore to provide adequate information for the reconstruction. A total of 122 TEM images were obtained from $-30^{\circ}$ to $+30^{\circ}$ at $1^{\circ}$ increments with a dual-axis model. The $3 \mathrm{D}$ structure of the nanopore was reconstructed by calculating the weighted back-projection of a series of oblique images with the IMOD software. Finally, the electronic tomographic image had an in-plane (X-Y) pixel size of approximately $2.6 \mathrm{~nm}$, and a size of approximately $0.35 \mathrm{~nm}$ along the $\mathrm{Z}$-axis. Based on experience in reconstructing $3 \mathrm{D}$ tomographic images, it is inferred that the $2 \mathrm{D}$ microscope image is related to the sidewall shape of the nanopore.

\section{Results and Discussion}

Figure 1(a) shows a schematic of the electron beam used to drill nanopores on a silicon nitride membrane which was subsequently observed with TEM. When the high-intensity $\left(10^{7}-10^{8} \mathrm{~A} \mathrm{~m}^{-2}\right)$ electron beam was used to drill nanopores on a silicon nitride membrane, a nanopore with an hourglass shape could be formed with sputtering $^{25-28}$. The use of the high-intensity electron beam for the illumination of the nanopore narrowed the sharp edge of the hourglass shape to a cylindrical shape, and resulted in the destruction of the original shape ${ }^{29}$. By contrast, a low-intensity electron beam $\left(-10^{6} \mathrm{~A} \mathrm{~m}^{-2}\right)$ could drill nanopores on a silicon nitride membrane without damaging the sidewall shape. Figure 1(b) shows an electron microscope image of a nanopore with a diameter of $15 \mathrm{~nm}$. The $3 \mathrm{D}$ reconstructed image based on the use of a series of oblique photographs of the nanopore obtained by electron tomography shows that the nanopore has an hourglass shape. When the nanopore diameter changed from 15 to $50 \mathrm{~nm}$ at increased drilling time, the low-intensity electron beam which was used to drill the nanopore on the silicon nitride membrane did not destroy the original structure but maintained the hourglass shape. This indicates that the low-intensity electron beam was less destructive to the silicon nitride membrane during the drilling process, as shown in Fig. 1(c,d). 
(a)

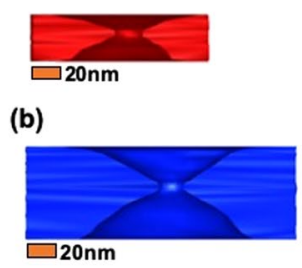

(c)

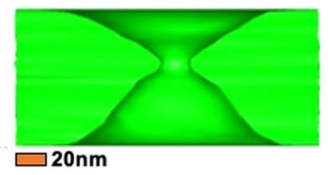

(d)

(e)

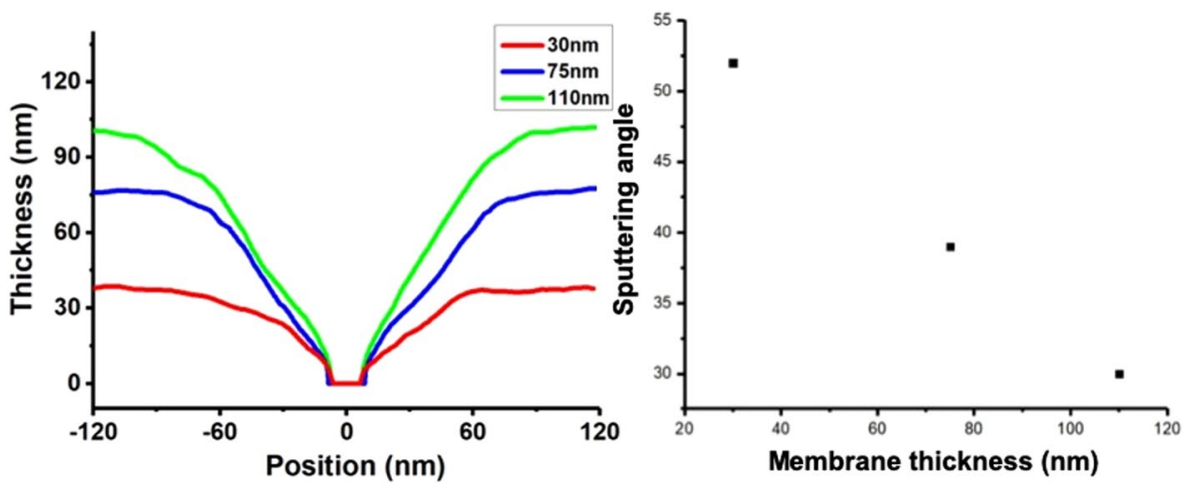

Figure 2. Reconstructed three-dimensional (3D) images of nanopores with diameters of $\sim 15 \mathrm{~nm} \mathrm{in} \operatorname{SiN}_{\mathrm{x}}$ membranes with thicknesses of (a) $110 \mathrm{~nm}$, (b) $75 \mathrm{~nm}$, and (c) $30 \mathrm{~nm}$. (d) Plots of thickness vs. position. (e) Plots of thickness of membrane vs. sputtering angle $\theta$.

To understand the relationship between the sputtering phenomenon and the thickness of the silicon nitride membrane, we used same electron beam conditions. Accordingly, the beam diameter was $5 \mathrm{~nm}$ and focused on the membrane during the nanopore drilling at different SiNx membrane thickness values. Figure 2(a-c) shows the focus beam drilled nanopore profile with 3D tomography. Figure 2(d) shows the correlation between the thickness and position using the reconstructed images. As the thickness and slope of the membrane increased, there was a correlation between the sputtering angle and membrane thickness. Figure 2(e) shows that the thicker membrane has a smaller sputtering angle $\theta$. The hourglass shape of the nanopore sidewall shape could be explained by the sputtering model equation ${ }^{30,31}$,

$$
E_{\mathrm{th}}=m_{0} c^{2}\left[\left(\frac{M E_{d}}{2 m_{0}{ }^{2} c^{2} \cos ^{2} \theta}+1\right)^{1 / 2}-1\right],
$$

where $m_{0}$ is the rest mass of the electron, $c$ is the speed of light, $M$ the atomic mass, $E_{d}$ is the displacement or binding energy of the atom, and $\theta$ is the angle between the trajectory of the displaced atom and the direction of the incident electron. The value of the displacement energy $\left(E_{d}\right)$ of the $\mathrm{SiN}_{\mathrm{x}}$ membrane is $16 \mathrm{eV}$, which corresponds to threshold electron energy $\left(E_{t h}\right)$ of approximately $180 \mathrm{keV}^{25}$. When the electron energy exceeded the threshold $E_{t h}$, it formed into an hourglass shape based on the sputtering on the anterior and posterior sides with the highest intensity of the electron beam applied at the center. The sputtering effect induced by the elastic scattering associated with the interactions among the electrons and atoms. The elastic scattering causes direct atomic displacement, such as the sputtering of the atoms ${ }^{25}$. Another mechanism of energy transfer from the incident electrons to the target material is radiolysis induced by the inelastic scattering ${ }^{32,33}$ associated with electron-electron interactions, resulting in the heating of the specimen ${ }^{34,35}$ and ionization. As the incident electron energy is increased, the effects of elastic scattering are increased, while those of inelastic scattering and heating are decreased. The main cause of the 3D sidewall shape of the nanopore was still dominated by the sputtering process.

Because sputtering was the main cause responsible for the formation of the sidewall shape and started from the center of the electron beam, the adjustment to the focal plane was equivalent to the adjustment of the center position of the electron beam. This resulted in the adjustment of the nanopore shape of the focal plane. When the low-intensity electron beam was used in drilling the nanopore within the focal plane in different heights and to maintain the same beam condition which included the beam's diameter and intensity, as shown in Fig. 3(a-f), it was found that the height of the narrow edge of the nanopore varied at different focal planes positions. This demonstrates that the hourglass shape associated with the beam profile depended upon whether the beam crossover or waist was inside or above the membrane. For drilling, we found that focus beams are more stable and made it easier to control the pore size compared with the defocus beam. Once a higher focal plane (defocus beam) is chosen for nanopore drilling, it is considerably much more difficult to control the drilled pore size. In conclusion, silicon nitride membrane damage from electron irradiation resulted in the direct atomic displacement that involved electron-atom scattering. When the impact of the electron-beam diameter on the drilling effect of silicon nitride membrane was investigated, electron beams with diameters of 20-100 $\mathrm{nm}$ were used. Changing the beam diameter from $20 \mathrm{~nm}$ to $100 \mathrm{~nm}$ would yield a beam intensity difference on the order of 10 . The intensity of the electron beam was in the range of approximately $10^{5}-10^{6} \mathrm{~A} / \mathrm{m}^{2}$, which was focused on the membrane upper surface. It shows that if the diameter of the electron beam increased, the membrane of the nanopore becomes thinner and flatter, as shown in Fig. 3(g-j). Figure 3(j) shows that when an electron beam with a diameter of $100 \mathrm{~nm}$ was used to illuminate a silicon nitride membrane with a thickness of $30 \mathrm{~nm}$ for approximately $30 \mathrm{~min}$, the membrane was relatively thin in comparison to the beginning. The underlying formation mechanism can be explained using the sputtering model, as shown in Fig. 4.

When an electron beam with a diameter of $100 \mathrm{~nm}$ was used to drill nanopores on a silicon nitride membrane with a thickness of $30 \mathrm{~nm}$, a small sputtering angle $\theta_{1}$ could form owing to the large thickness of the 
(a)

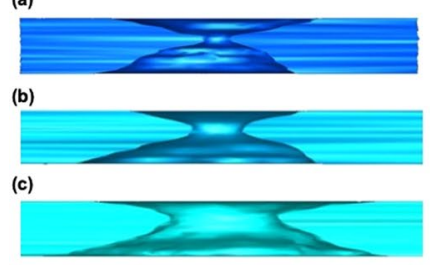

(d)

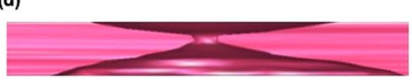

(e)

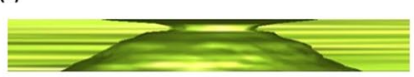

(f)

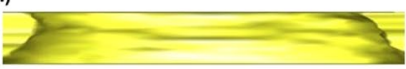

$20 \mathrm{~nm}$

$20 \mathrm{~nm}$

$20 \mathrm{~nm}$

$20 \mathrm{~nm}$

$20 \mathrm{~nm}$

20nm

$\stackrel{20 \mathrm{~nm}}{\square}$

$20 \mathrm{~nm}$

$\square$

$20 \mathrm{~nm}$

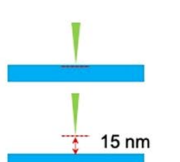

$\frac{1}{7} 40 \mathrm{~nm}$
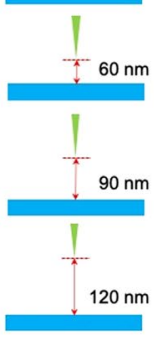

(g)

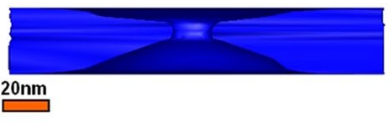

(h)

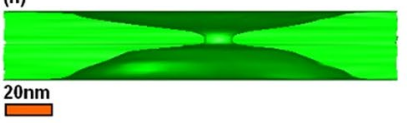

(i)

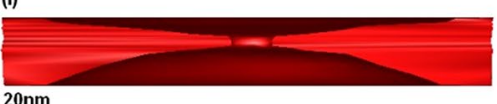

$20 \mathrm{~nm}$

(j)

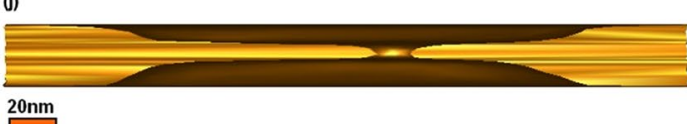

Figure 3. Reconstructed 3D images of nanopores. Use of different focal plane positions of nanopores drilled with electron-beams in a membrane with a thickness of $30 \mathrm{~nm}$. Electron beam is focused with different heights, as follows: (a) 0, (b) 15, (c) 40, (d) 60, (e) 90 and (f) $120 \mathrm{~nm}$. Reconstructed 3D image of nanopore sculpted by extended electron beam with diameters of (g) 20, (h) 40, (i) 80 , and (j) $100 \mathrm{~nm}$.

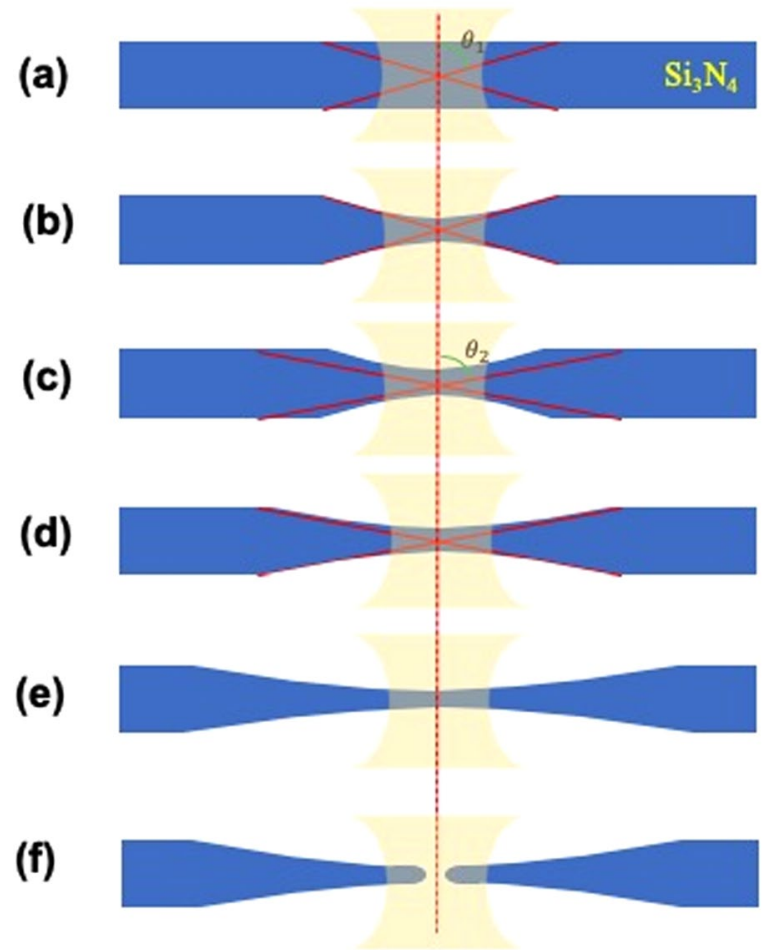

Figure 4. Schematic of nanopore drilling process using an electron beam with a diameter of $100 \mathrm{~nm}$.

Membrane thickness: $30 \mathrm{~nm}$, beam intensity: $10^{6} \mathrm{~A} \mathrm{~m}^{-2}$, Focal plane: On the membrane surface. (a) Drilling of the $\mathrm{SiN}_{\mathrm{x}}$ membrane with an electron beam with a diameter of $100 \mathrm{~nm}$. The thickness of the membrane is large at the beginning, so the sputtering angle is relatively small. (b) Membrane thinning following the sputtering of the atoms. (c) Electron beam drilling is continually used, and the sputtering angle $\theta_{2}$ is larger than $\theta_{1}$ owing to the thinning of the membrane. (d) The membrane thickness decreases even more by the sputtering. (e) This cycle is repeated, eventually resulting in a flat and thin membrane. (f) Flat and thin membrane with a nanopore with an hourglass sidewall shape.

membrane. The membrane became thinner after the atoms were sputtered. When the electron beam was continually used to drill nanopores, the thickness of the membrane was reduced and resulted in a larger sputtering angle $\theta_{2}$. Correspondingly, the thickness of the membrane was further decreased by sputtering. This cycle was repeated until a flattened and thin membrane eventually formed with an hourglass-shaped nanopore. A flat and 
(a)

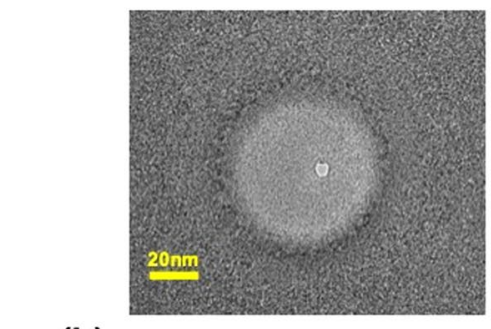

(b)

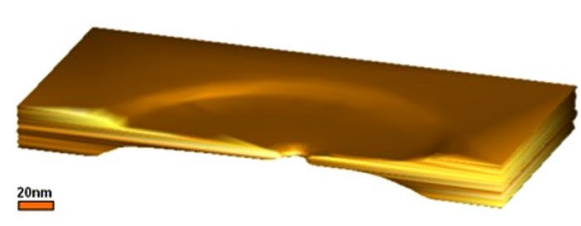

(c)

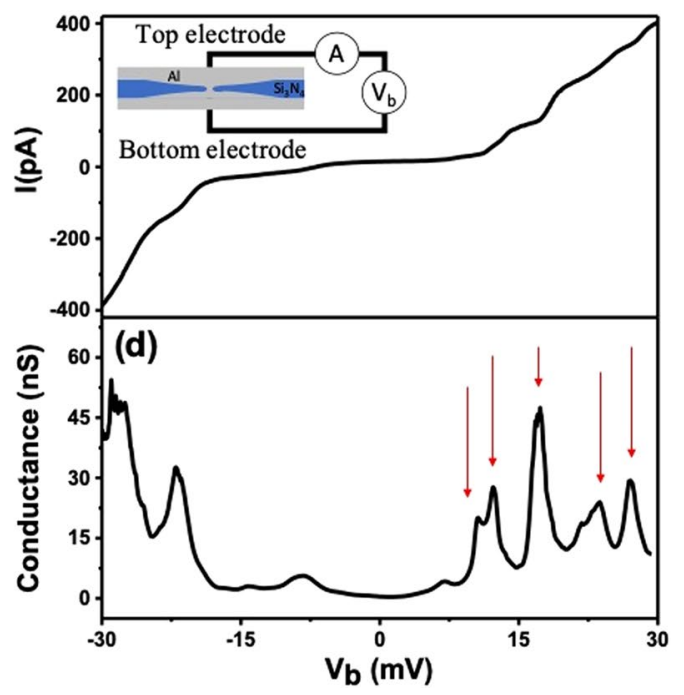

Figure 5. (a) Transmission electron microscopy (TEM) image of a flattened hourglass-shaped nanopore (b) Reconstructed 3D image of a flattened hourglass-shaped nanopore (c) $I-V$ curve of device at $T=4.2 \mathrm{~K}$. Inset: schematic of measurement setup of metallic quantum-dot device. (d) Differential conductance $(d I / d V)$ as a function of bias voltage $V_{b}$ at $4.2 \mathrm{~K}$. The arrows denote the discrete electric states of quantum dot.

thin membrane with an hourglass-shaped nanopore is a better choice for DNA sequencing. This is attributed to the fact that when the DNA sequence passes through the nanopore, a smaller thickness which provides a shorter transport length critically affects the translocation speed and measured signal resolution. One base pair of length along the strand is approximately $0.34 \mathrm{~nm}$. A smaller membrane thickness results in a higher resolution for DNA sequencing ${ }^{36-38}$.

In addition to their applications in DNA sequencing experiments, a flattened hourglass-shaped nanopore can be used as a template for nanoscale electronic devices (see Fig. 5(a,b)), such as quantum-dot devices. To verify the conduction characteristics of aluminum used in the following experiments, preliminary experiments were carried out using a typical hourglass-shaped nanopore with a radius of $60 \mathrm{~nm}$ as a template (see Fig. S5 of Supplementary Material). The top part of the silicon nitride membrane of the flat nanopore in our experiment was vapor-deposited with $60 \mathrm{~nm}$ of aluminum to construct the upper electrode, and $250 \mathrm{~nm}$ of aluminum was vapor-deposited at the bottom to construct the lower electrode. A sandwich-structure device with a metallic disc was eventually achieved, as shown in the inset of Fig. 5(c). The experiments included the measurement of the tunneling current which flowed vertically from the upper electrode, via the aluminum's nanoconstrictions, to the lower electrode. The samples were characterized by the measurement of the electrical transport properties at $4.2 \mathrm{~K}$. Figure $5(\mathrm{c})$ shows the current-voltage $(I-V)$ characteristics of the flat nanopore device. The $I-V$ curve shows a suppression of the current at low voltages owing to the Coulomb blockade and stepwise increase of the current owing to the discrete energy spectrum. Additional step increases occur when the voltage is raised sufficiently to allow tunneling via other, high-energy discrete energy levels. Thus, a plot of differential conductance as a function of the bias voltage $\left(V_{b}\right)$ displays a series of peaks, as shown in Fig. 5(d). A large gap is observed, followed by a series of peaks associated with tunnelling into the excited states of the metallic quantum dots. This is an important experimental tool for investigating quantum dots where a gate electrode cannot be defined.

The spectrum of discrete electric states shown in Fig. 5(c,d) can be explained by the theory of metallic quantum dots $s^{39,40}$. When an electron enters the nanoconstriction region of the nanopore, an additional, nonzero energy can block the tunneling of electrons. If the applied bias voltage provides adequate energy to surpass the charge energy and overcome the discrete energy level difference, the electron can tunnel from the upper electrode on the dot. At this time, the electrochemical potential of the quantum dot will be enhanced, and the next electron will be blocked from the quantum dot. This phenomenon is called Coulomb blockade. The Coulomb blockade can be removed by changing the bias voltage. When the external bias voltage is further increased, and the energy corresponds to the discrete zero-dimensional (0D) states of the quantum dot, the Coulomb staircase effect can be observed. Accordingly, the position of the staircase will correspond to the positions of the $0 \mathrm{D}$ states. The structure of the device is similar to metallic point contact, but the experimental results have not observed the conductance quantization ${ }^{41}$. We provided an explanation based on which the deposition of the aluminum on this nanopore structure will form an electrically isolated particle rather than a metallic point contact. The conductance of the isolated particle can be measured by attaching it to electrodes via high-resistance tunnel junctions. From the positions of the voltage thresholds for steps in the $I-V$ characteristics, the capacitances of the tunnel junction within the device can be determined directly ${ }^{42}$. The electrode-to-particle capacitances are $C_{1}=10.6 \mathrm{aF}$ and $C_{2}=8.5 \mathrm{aF}$, and the charging energy is $4.2 \mathrm{meV}$. Based on the electrode-to-particle capacitances, the size of the particle can be roughly estimated; the radius of the particle is $3.0-3.4 \mathrm{~nm}$ by assuming it is spherical for the device ${ }^{39}$. In addition, the device has high-resistance tunneling junctions that provide weak coupling to the environment, the discrete 
electronic states can be observed. Finally, a simple sandwich structure constructed on this methodology enabled us to easily observe the behaviors of the metallic quantum-dot device. This device is expected to become a niche area in applications of quantum computing ${ }^{43}$.

\section{Conclusions}

In summary, we demonstrated the low-intensity electron beam technique used to drill nanopores on a silicon nitride membrane, and exploited electron tomography to investigate the formation mechanism of the nanopore. We found that the low-intensity electron beam could maintain the nanopore's shape without destroying its structure. When the focal plane of the electron beam was adjusted, the height of the narrow region of the nanopore changed. Accordingly, while the diameter of the electron beam was altered, the thinner membrane with the nanopore could be manipulated. The low-intensity electron beam had high elasticity, low destructiveness, and could also flexibly construct the shape of the desired 3D sidewall. Moreover, we presented a simple, versatile technique for the fabrication of quantum devices. This technology was used in conjunction with nanopore drilling and coating technologies to prepare metallic quantum-dot devices, and could be applied to the development of quantum electronics in the future.

Received: 12 September 2019; Accepted: 25 November 2019;

Published online: 09 December 2019

\section{References}

1. Li, J., Gershow, M., Stein, D., Brandin, E. \& Golovchenko, J. A. DNA molecules and configurations in a solidstate nanopore microscope. Nature Mater 2, 611 (2003).

2. Sze, J. Y. Y., Ivanov, A. P., Cass, A. E. G. \& Edel, J. B. Single molecule multiplexed nanopore protein screening in human serum using aptamer modified DNA carriers. Nat. Commun. 8, 1552 (2017).

3. Traversi, F. et al. Detecting the translocation of DNA through a nanopore using graphene nanoribbons. Nat. Nanotechnol. 8, 939 (2013).

4. Feng, J. et al. Electrochemical Reaction in Single Layer MoS2: Nanopores Opened Atom by Atom. Nano Lett 15, 3431 (2015).

5. Heerema, S. J. et al. Probing DNA Translocations with Inplane Current Signals in a Graphene Nanoribbon with a Nanopore. ACS Nano 12, 2623 (2018)

6. Shi, X., Verschueren, D. V. \& Dekker, C. Active Delivery of Single DNA Molecules into a Plasmonic Nanopore for Label-Free Optical Sensing. Nano Lett. 18, 8003 (2018).

7. Wang, Y. et al. Fabrication of multiple nanopores in a $\mathrm{SiN}_{\mathrm{x}}$ membrane via controlled breakdown. Sci. Rep. 8, 1234 (2018).

8. Faramarzi, V., Ahmadi, V., Fotouhi, B. \& Abasifard, M. A potential sensing mechanism for DNA nucleobases by optical properties of GO and MoS2 Nanopores. Sci. Rep. 9, 6230 (2019).

9. Yanagi, I., Ishida, T., Fujisaki, K. \& Takeda, K. Fabrication of 3-nm-thick $\mathrm{SiN}_{\mathrm{x}}$ membranes for solid-state nanopores using the poly-Si sacrificial layer process. Sci. Rep. 5, 14656 (2015).

10. Yanagi, I., Fujisaki, K., Hamamura, H. \& Takeda, K. Thickness-dependent dielectric breakdown and nanopore creation on sub-10nm-thick SiN membranes in solution. J. Appl. Phys. 121, 45301 (2017).

11. Spinney, P. S., Howitt, D. G., Smith, R. L. \& Collins, S. D. Nanopore formation by low-energy focused electron beam machining. Nanotechnology 21, 375301 (2010).

12. Verschueren, D. V., Yang, W. \& Dekker, C. Lithography-based fabrication of nanopore arrays in freestanding SiN and graphene membranes. Nanotechnology 29, 145302 (2018).

13. Yemini, M., Hadad, B., Liebes, Y., Goldner, A. \& Ashkenasy, N. The controlled fabrication of nanopores by focused electron-beaminduced etching. Nanotechnology 20, 245302 (2009).

14. Shim, J. et al. Detection and Quantification of Methylation in DNA using Solid-State Nanopores. Sci. Rep. 3, 1389 (2013).

15. Wu, M. J., Krapf, D., Zandbergen, M., Zandbergen, H. \& Batson, P. E. Formation of nanopores in a $\mathrm{SiNSiO}_{2} \mathrm{membrane}$ with an electron beam. Appl. Phys. Lett. 87, 113106 (2005).

16. Hout, M. et al. Controlling nanopore size, shape and stability. Nanotechnology 21, 115304 (2010).

17. Liu, S. et al. Controlled deformation of $\mathrm{SiN}_{\mathrm{x}}$ nanopores using focused electron beam in a transmission electron microscope, Nanotechnology 22, 115302 (2011).

18. Storm, J., Chen, J. H., Ling, X. S., Zandbergen, H. W. \& Dekker, C. Fabrication of solid-state nanopores with single-nanometre precision. Nature Materials 2, 537 (2003).

19. Akahori, R. et al. Slowing single-stranded DNA translocation through a solid-state nanopore by decreasing the nanopore diameter. Nanotechnology 25, 275501 (2014).

20. Wanunu, M., Sutin, J., McNally, B., Chow, A. \& Meller, A. DNA Translocation Governed by Interactions with Solid-State Nanopores. Biophys. J. 95, 4716-4725 (2008).

21. Wu, M. Y. et al. Control of Shape and Material Composition of Solid-State Nanopores. Nano Lett. 9, 479 (2009).

22. Thomasn, J. M. et al. Nanoscale electron tomography and atomic scale high-resolution electron microscopy of nanoparticles and nanoclusters: A short survey. Prog. Nat. Sci.: Mater. Int. 23, 222-234 (2013).

23. Kremer, J. R., Mastronarde, D. N. \& McIntosh, J. R. Computer Visualization of Three-Dimensional Image Data Using IMOD. J. Struct. Biol. 116, 71 (1996).

24. Kim, M. J. et al. Rapid fabrication of uniformly sized nanopores and nanopore arrays for parallel DNA analysis. Advanced Materials 18, 3149-3153 (2006).

25. Kim, H. M., Lee, M. H. \& Kim, K. B. Theoretical and experimental study of nanopore drilling by a focused electron beam in transmission electron microscopy. Nanotechnology 22, 275303 (2011).

26. Qian, H. \& Egerton, R. F. Solid-state nanopores of controlled geometry fabricated in a transmission electron microscope. Appl. Phys. Lett. 111, 193106 (2017).

27. Jiang, Z., Mihovilovic, M., Chan, J. \& Stein, D. Fabrication of nanopores with embedded annular electrodes and transverse carbon nanotube electrodes. J. Phys.: Condens. Matter 22, 454114 (2010).

28. Chen, S. J. et al. The applications of in situ electron energy loss spectroscopy to the study of electron beam nanofabrication. Microsc. Microanal. 15(03), 204-212 (2009).

29. Kim, M. J., McNally, B., Murata, K. \& Meller, A. Characteristics of solid-state nanometre pores fabricated using a transmission electron microscope. Nanotechnology 18, 115304 (2007).

30. Spinney, P. S., Howitt, D. G., Collins, S. D. \& Smith, R. L. Electron beam stimulated oxidation of carbon. Nanotechnology 20, 465301 (2009).

31. Howitt, D. G. The electron beam hole drilling of silicon nitride thin films. Journal of Applied Physics 103, 024310 (2008).

32. Egerton, R. F., Li, P. \& Malac, M. Radiation damage in the TEM and SEM. Micron 35, 399-409 (2004). 
33. Levine, Z. H. Tomography in the multiple scattering regime of the scanning transmission electron microscope. Applied Physics Letters 82, 3943-3945 (2003).

34. Yokota, T., Murayama, M. \& Howe, J. M. Theoretical and experimental study of nanopore drilling by a focused electron beam in transmission electron microscopy. Phys. Rev. Lett. 91, 265504 (2003).

35. Bysakh, S., Shimojo, M. K. \& Furuya, K. J. Mechanisms of nano-hole drilling due to nano-probe intense electron beam irradiation on a stainless steel. J. Vac. Sci. Technol. B 22, 2620-7 (2004).

36. Meni, W. et al. Rapid electronic detection of probe-specifc micrornas using thin nanopore sensors. Nature Nanotechnology $\mathbf{5}$, 807-814 (2010).

37. Spinney, P. S., Collins, S. D., Howitt, D. G. \& Smith, R. L. Fabrication and Characterization of a Solid State Nanopore with SelfAligned Carbon Nanoelectrodes for Molecular Detection. Nanotechnology 23, 135501 (2012).

38. Gierhart, B. C. et al. Nanopore with transverse nanoelectrodes for electrical characterization and sequencing of DNA. Sens. Actuators, B 132, 593 (2008).

39. Ralph, D. C., Black, C. T. \& Tinkham, M. Spectroscopic Measurements of Discrete Electronic States in Single Metal Particles. Phys. Rev. Lett. 74, 3241 (1995).

40. Black, C. T., Ralph, D. C. \& Tinkham, M. Spectroscopy of the Superconducting Gap in Individual Nanometer-Scale Aluminum Particles. Phys. Rev. Lett. 76, 688 (1996).

41. Scheer $e t$ al. The signature of chemical valence in the electrical conduction through a single-atom contact. Nature 394, 154 (1998).

42. Hanna, A. E. \& Tinkham, M. Variation of the Coulomb staircase in a two-junction system by fractional electron charge. Phys. Rev. B. 44, 5919 (1991).

43. Loss, D. \& DiVincenzo, D. P. Quantum computation with quantum dots. Phys. Rev. A 57, 120 (1998)

\section{Acknowledgements}

Productive discussions with Guang-Yin Chen, Rui-Zhi Su, Tsan-Chu Lin, and Watson Kuo, are acknowledged. The authors would like to thank the Ministry of Science and Technology of Taiwan for financially supporting this work under Project numbers MOST 106-2112-M-018-004, MOST 105-2731-M-018-001, and MOST 106-2731-M-018-001. This work has been supported in part by the Nanoscience and Technology Center at the National Changhua University of Education.

\section{Author contributions}

Chien-Han Chen measured the low-temperature electrical data, analyzed the 3D images, and drew all figures. $\mathrm{Xu}$-yan Chang fabricated the devices and reconstructing the 3D images. Cen-Shawn Wu designed the study, set up the measurement system and wrote the manuscript. Cen-Shawn Wu also supervised all portions. All authors reviewed the manuscript.

\section{Competing interests}

The authors declare no competing interests.

\section{Additional information \\ Supplementary information is available for this paper at https://doi.org/10.1038/s41598-019-55190-y.}

Correspondence and requests for materials should be addressed to C.-S.W.

Reprints and permissions information is available at www.nature.com/reprints.

Publisher's note Springer Nature remains neutral with regard to jurisdictional claims in published maps and institutional affiliations.

(c) (i) Open Access This article is licensed under a Creative Commons Attribution 4.0 International

License, which permits use, sharing, adaptation, distribution and reproduction in any medium or format, as long as you give appropriate credit to the original author(s) and the source, provide a link to the Creative Commons license, and indicate if changes were made. The images or other third party material in this article are included in the article's Creative Commons license, unless indicated otherwise in a credit line to the material. If material is not included in the article's Creative Commons license and your intended use is not permitted by statutory regulation or exceeds the permitted use, you will need to obtain permission directly from the copyright holder. To view a copy of this license, visit http://creativecommons.org/licenses/by/4.0/.

(c) The Author(s) 2019 